\title{
INVARIANTS FOR FAMILIES OF BRIESKORN VARIETIES
}

\author{
TERRY LAWSON
}

\begin{abstract}
Fintushel and Stern have defined invariants for certain homology 3spheres which, if positive, show that the homology 3-sphere cannot bound a positive definite 4-manifold with no 2-torsion in its first homology. In this note a numbertheoretic formula is given for these invariants. It is used to show that all members of certain families of Brieskorn varieties have the same invariants, and hence exhibit the same nonbounding when one of the invariants is positive.
\end{abstract}

1. A formula for the Fintushel-Stern invariant. In [FS1] an invariant $R\left(a_{1}, \ldots, a_{n}\right)$ was defined for a Brieskorn variety $M\left(a_{1}, \ldots, a_{n}\right)$ and was used to show that when this invariant was positive the Brieskorn variety could not bound a positive definite 4-manifold with no 2-torsion in its first homology. In [FL] the invariant was extended for other Euler classes to an invariant $R\left(a_{1}, \ldots, a_{n} ; l\right)$ and it was shown that if this invariant is positive for some $l$ and certain additional conditions are satisfied, then the same conclusion holds. In the course of investigating the application of this extended invariant, we noticed a pattern in the examples which occurred. This suggested that for small enough $l$, the invariant $R$ is constant on a family of Brieskorn varieties where the last entry is changed by adding multiples of the product of the other entries, i.e., $a_{n}^{\prime}=a_{n}+k b_{n}$, where $b_{n}=a_{1} \cdots a_{n-1}$. In this note we will prove this result by giving a number-theoretic formula for the cotangent sums which are part of the index $R$. This formula can be considered a generalization of the formula given for the index when $l=1$ in $[\mathbf{N Z}]$. In fact our argument will follow their argument closely. We will also note some properties of the $\mu$ invariant on these families.

We first review the computational methods given in [NZ]. Associated with $M\left(a_{1}, \ldots, a_{n}\right)$ are pairs $\left(a_{i}, b_{i}\right)$, where $b_{i}$ is the product of the $a_{j}$ with $j \neq i$. There is a unique number $b_{i}^{*}$ with $0<b_{i}^{*}<a_{i}$ so that $b_{i} b_{i}^{*} \equiv 1 \bmod a_{i}$. Define $b_{L}=\sum b_{i}^{*} / a_{i}$ $-1 / \alpha$, where $\alpha$ is the product of all the $a_{i}$. Note $1 \leqslant b_{L} \leqslant n-1$. This will be the middle number in a plumbing diagram with all positive entries (and signature $=$ rank -2$)$. It is related to the middle number $-b_{N Z}$ of the negative definite plumbing diagram given in [NZ] by $b_{N Z}=n-b_{L}$. In terms of our $b_{L}$, their formula for $R$ when $l=1$ becomes $(2 n-3)-2 b_{L}$.

Our general formula will be $R\left(a_{1}, \ldots, a_{n}, l\right)=(n-3)-2 b_{L} l^{2}+\sum N\left(a_{i}, b_{i} ; l\right)$, where $N\left(a_{i}, b_{i} ; l\right)$ will involve solutions to congruences mod $a_{i}$ and divisibility by $a_{i}$.

Received by the editors November 25, 1985.

1980 Mathematics Subject Classification (1985 Revision). Primary 57R90, 55R55.

Partially supported by a Tulane University Committee on Research grant.

(C)1987 American Mathematical Society $0002-9939 / 87 \$ 1.00+\$ .25$ per page 
When $\operatorname{gcd}(a, b)=1$ define $[a, b ; k]$ to be 1 if one of $b, 2 b, \ldots,\left(b^{*}-1\right) b \equiv k$ $\bmod a$ and 0 otherwise, where $0<b^{*}<a$ and $b b^{*} \equiv 1 \bmod a$. Note that $r b \equiv k+1$ $\bmod a, 1 \leqslant r \leqslant b^{*}-1$ iff $\left(b^{*}-r\right) b \equiv-k \bmod a, 1 \leqslant b^{*}-r \leqslant b^{*}-1$; thus $[a, b ; k+1]=[a, b ;-k]$.

Define $[a \mid k]$ to be 1 if $a$ divides $k$ and 0 otherwise. If $l=1$, define $N(a, b ; l)=1$. If $l>1$, define

$$
\begin{aligned}
N(a, b ; l)= & (2 l-1)+2 \sum_{k=1}^{l-1}(2 l-1-2 k)[a, b ; k+1] \\
& +[a \mid l]+\sum_{k=2}^{l-1} 4(l-k)[a \mid k] .
\end{aligned}
$$

In order to prove this formula, first recall the formula

$$
\begin{aligned}
& R\left(a_{1}, \ldots, a_{n} ; l\right) \\
& =(n-3)+\frac{2 l^{2}}{\alpha}+\sum_{i=1}^{n} \frac{2}{a_{i}} \sum_{k=1}^{a_{i}-1} \cot \left(\pi b_{i} k / a_{i}\right) \cot \left(\pi k / a_{i}\right) \sin ^{2}\left(\pi k l / a_{i}\right) .
\end{aligned}
$$

We will now concentrate on reexpressing this last contangent sum. Following the notation of [FS, §10], let us call

$$
\delta(a ; r, s ; l)=\frac{2}{a} \sum_{k=1}^{a-1} \cot (\pi k r / a) \cot (\pi k s / a) \sin ^{2}(\pi k l / a)
$$

and $\delta(a ; r, s)=\delta(a ; r, s ; 1)$. Note that

(1) $\delta(a ; r, s ; l)=\delta\left(a, r s^{*}, 1, s^{*} l\right)$ by reindexing. Thus the computation of the cotangent sums in $R(X, e)$ in [FS1] will follow from our formula below for the case where $s=1$.

(2) $\delta(a ; r, r)=\delta(a ; r, r ; 1)=\delta\left(a ; 1,1, r^{*}\right)$

(3) $\delta(a ; r, s ; l)$ only depends on $r, s, l \bmod a$.

Proposition. $\delta(a ; b, 1 ; l)=N(a, b ; l)-2 b^{*} l^{2} / a$.

Proof. As in [NZ] one makes the substitution $\tau=\exp (2 \pi i x)$, so $\cot (\pi x)=$ $i(\tau+1) /(\tau-1)$ and $\sin ^{2} \pi x=-\frac{1}{4} \tau^{-1}(\tau-1)^{2}$. Thus

$$
\begin{aligned}
& \delta(a ; b, 1 ; l)=\frac{2}{a} \sum_{k=1}^{a-1} \cot \left(\frac{\pi b k}{a}\right) \cot \left(\frac{\pi k}{a}\right) \sin ^{2}\left(\frac{\pi k l}{a}\right) \\
& =\frac{1}{2 a} \sum_{\substack{\tau^{a}=1 \\
\tau \neq 1}} \frac{\left(\tau^{b+1}\right)}{\left(\tau^{b}-1\right)} \frac{\tau+1}{\tau-1} \tau^{-l}\left(\tau^{l}-1\right)^{2} \\
& =\frac{1}{2 a} \sum_{\substack{\tau^{a}=1 \\
\tau \neq 1}}\left(\tau^{b}+1\right)(\tau+1) \tau^{-1} \tau^{-(l-1)}\left(1+\tau+\cdots+\tau^{(l-1)}\right)^{2} \frac{\tau-1}{\tau^{b}-1}
\end{aligned}
$$




$$
\begin{aligned}
= & \frac{1}{2 a} \sum_{\substack{\tau^{a}=1 \\
\tau \neq 1}}\left(\tau^{b}+\tau^{b-1}+1+\tau^{-1}\right)\left(1+\tau^{b}+\tau^{2 b}+\cdots+\tau^{\left(b^{*}-1\right) b}\right) \\
& \times\left(1+\tau+\cdots+\tau^{(l-1)}\right)\left(1+\tau^{-1}+\cdots+\tau^{-(l-1)}\right) \\
= & \frac{1}{2 a} \sum_{\tau^{a}=1}\left(\tau^{b}+\tau^{b-1}+1+\tau^{-1}\right)\left(1+\tau^{b}+\cdots+\tau^{\left(b^{*}-1\right) b}\right) \\
& \times\left(1+\tau+\cdots+\tau^{(l-1)}\right)\left(1+\tau^{-1}+\cdots+\tau^{-(l-1)}\right)-\frac{2}{a} b^{*} l^{2} \\
= & \frac{1}{2 a} \sum_{\tau^{a}=1}\left(\tau^{b}+\tau^{b-1}+1+\tau^{-1}\right)\left(1+\tau^{b}+\cdots+\tau^{\left(b^{*}-1\right) b}\right) \\
& \times\left(l+(l-1)\left(\tau+\tau^{-1}\right)+\cdots+\left(\tau^{(l-1)}+\tau^{-(l-1)}\right)\right)-\frac{2}{a} b^{*} l^{2} .
\end{aligned}
$$

We will separate off the last term and concentrate on showing the sum above is $N(a, b ; l)$.

We use the fact that

$$
\sum_{\tau^{a}=1} \tau^{x}= \begin{cases}a & \text { if } a \mid x \\ 0 & \text { otherwise }\end{cases}
$$

to evaluate $(1 / 2 a) \sum_{\tau^{a}=1} \tau^{k}\left(\tau^{b}+\tau^{b-1}+1+\tau^{-1}\right)\left(1+\tau^{b}+\cdots+\tau^{\left(b^{*}-1\right) b}\right)$. We write this as four separate terms.

$$
\frac{1}{2 a} \sum_{\tau^{a}=1} \tau^{b+k}\left(1+\tau^{b}+\cdots+\tau^{\left(b^{*}-1\right) b}\right)=\frac{1}{2}([a, b ;-k]+[a \mid k+1])
$$

(since $a$ divides one of $b+k, 2 b+k, \ldots,\left(b^{*}-1\right) b+k$ iff one of $b, 2 b, \ldots,\left(b^{*}-1\right) b \equiv-k \bmod a$ and $a \mid b^{*} b+k=\left(b^{*} b-1\right)+$ $(k+1)$ iff $a \mid k+1)$.

Similarly,

(2) $\frac{1}{2 a} \sum_{\tau^{a}=1} \tau^{k}\left(1+\tau^{b}+\cdots+\tau^{\left(b^{*}-1\right) b}\right)=\frac{1}{2}([a, b ;-k]+[a \mid k])$.

(3) $\frac{1}{2 a} \sum_{\tau^{a}=1} \tau^{b+k-1}\left(1+\tau^{b}+\cdots+\tau^{\left(b^{*}-1\right) b}\right)=\frac{1}{2}([a, b ; 1-k]+[a \mid k])$.

(4) $\frac{1}{2 a} \sum_{\tau^{a}=1} \tau^{k-1}\left(1+\tau^{b}+\cdots+\tau^{\left(b^{*}-1\right) b}\right)=\frac{1}{2}([a, b ; 1-k]+[a \mid k-1])$.

Thus

$$
\begin{aligned}
\frac{1}{2 a} \sum_{\tau^{a}=1} \tau^{k} & \left(\tau^{b}+\tau^{b-1}+1+\tau^{-1}\right)\left(1+\tau^{b}+\cdots+\tau^{\left(b^{*}-1\right) b}\right) \\
= & \frac{1}{2}(2[a, b ;-k]+2[a, b ; 1-k]+[a \mid k-1]+2[a \mid k]+[a \mid k+1]) .
\end{aligned}
$$


The sum then becomes

$$
\begin{aligned}
\frac{1}{2}(2 l & +(l-1)(2[a, b ;-1]+1+[a \mid 2]+2[a, b ; 2]+[a \mid 2]+1) \\
+ & (l-2)(2[a, b ;-2]+2[a, b ;-1]+2[a \mid 2]+[a \mid 3] \\
& +2[a, b ; 2]+2[a, b ; 3]+[a \mid 3]+2[a \mid 2]) \\
+ & (l-3)(2[a, b ;-3]+2[a, b ;-2]+[a \mid 2]+2[a \mid 3] \\
& +[a \mid 4]+2[a, b ; 3]+2[a, b ; 4]+[a \mid 4]+2[a \mid 3]+[a \mid 2])+\cdots \\
& +(2[a, b ; 1-l]+2[a, b ; 2-l]+[a \mid l-2] \\
& +2[a \mid l-1]+[a \mid l]+2[a, b ; l]+[a, l]+2[a \mid l-1]+[a \mid l-2])) \\
= & (2 l-1)+(2 l-3)([a, b ;-1]+[a, b ; 2])+(2 l-5)([a, b ;-2]+[a, b ; 3]) \\
& +\cdots+([a, b ;-(l-1)]+[a, b ; l])+4(l-2)[a \mid 2] \\
& +4(l-3)[a \mid 3]+\cdots+4[a \mid(l-1)]+[a \mid l] \\
= & (2 l-1)+\sum_{k=1}^{l-1}(2 l-1-2 k)([a, b,-k]+[a, b ; k+1]) \\
& +[a \mid l]+\sum_{k=2}^{l-1} 4(l-k)[a \mid k] . \\
= & N(a, b ; l) .
\end{aligned}
$$

Corollary 1. $R\left(a_{1}, \ldots, a_{n} ; l\right)=(n-3)-2 b_{L} l^{2}+\sum N\left(a_{i} ; b_{i} ; l\right)$

Proof. This follows from the Proposition and $2 l^{2} / \alpha-\sum 2 b_{i}^{*} l^{2} / a_{i}=-2 b_{L} l^{2}$.

The following corollary contains some computationally useful facts (cf. [FS1, FS2, L]) which follow immediately from the Proposition.

COROLlaRY 2. (a) If $l<a$,

$$
\delta(a ; b, 1, l)=(2 l-1)+2 \sum_{k=1}^{l-1}(2 l-1-2 k)[a, b ; k+1]-2 b^{*} l^{2} / a .
$$

In particular, if $b=1, \delta(a ; 1,1 ; l)=(2 l-1)-2 l^{2} / a$. If $(a, l)=1$, then $\delta\left(a, l^{*}, l^{*}\right)=\delta(a ; 1,1, l)$.

(b) $\delta(a ; r, s)=\delta\left(a ; r s^{*}, 1, s^{*}\right) \equiv-2 r^{*} s^{*} / a \bmod \mathbf{Z}$.

Remark. Here is a way to compute $\sum_{k=1}^{l-1}(2 l-1)[a, b ; k+1]$. Compute $b$, $2 b, \ldots,\left(b^{*}-1\right) b \bmod a$. Let $n_{1}, \ldots, n_{p}$ be the ones which are $\leqslant l$. Then

$$
\sum_{k=1}^{l-1}(2 l-1-2 k)[a, b ; k+1]=p(2 l+1)-2\left(n_{1}+\cdots+n_{p}\right) .
$$

2. Application to families of Brieskorn varieties. Now let us consider a family $M\left(a_{1}, \ldots, a_{n-1}, a_{n}+p a_{1} \cdots a_{n-1}\right)$ of Brieskorn varieties. We will apply the formula above to show that if $l<a_{n}$, then all members of the family will have the same invariant $R$. Note that replacing $a_{n}$ by $a_{n}^{\prime}$ does not change $\left(\bmod a_{i}\right)$ any of the terms $b_{i}$ and $b_{i}^{*}$ for $i<n$. For $i=n, b_{n}^{\prime}=b_{n}$. We claim $\left[a_{n}, b_{n} ; k\right]=\left[a_{n}^{\prime}, b_{n}^{\prime} ; k\right]$ for $2 \leqslant k<a_{n}$. This will follow from showing $[a, b ; k]=[a+b, b ; k]$ for $2 \leqslant k<a$. 
We write $b b^{*}=m a+1$. Then $b\left(b^{*}+m\right)=m(a+b)+1$ so $b^{* *}=b^{*}+m$ satisfies $b b^{* *} \equiv 1 \bmod (a+b)$. Now suppose $[a, b ; k]=1$ for $2 \leqslant k \leqslant a-1$. Then there is an $x, 1 \leqslant x \leqslant\left(b^{*}-1\right)$ so that $b x=y a+k$. Then $x \leqslant b^{*}-1$ implies $y<m$. Note $b(x+y)=y(a+b)+k$ and $x+y \leqslant\left(b^{*}-1\right)+m=b^{* *}-1$. Thus $[a+b, b ; k]=1$.

Conversely, suppose $[a+b, b ; k]=1,2 \leqslant k \leqslant a-1$. Then there is an $x$ with $1 \leqslant x \leqslant b^{* *}-1=b^{*}+m-1$ with $b x=y(a+b)+k$. Then $b(x-y)=y a+k$. Since the right-hand side is positive, $1 \leqslant x-y$. Again $1 \leqslant x<b^{*}+m$ implies $y<m$, and thus $y a+k<m a$. We claim $x-y \leqslant b^{*}-1$. For if $x-y \geqslant b^{*}$, this implies $x \geqslant y+b^{*}$. Hence $y(a+b)+k=x b \geqslant\left(y+b^{*}\right) b=m a+1+y b$. Subtracting $y b$ from both sides gives $y a+k \geqslant m a$, a contradiction. Thus $1 \leqslant x-y \leqslant$ $b^{*}-1$ and so $[a, b ; k]=1$. Thus if $l<a$, the terms $\left[a_{n}, b_{n} ; k+1\right]$ in the sum for $N\left(a_{n}, b_{n} ; l\right)$ will be unchanged upon replacing $a_{n}$ by $a_{n}^{\prime}$. The terms $\left[a_{n} \mid k\right]$ and $\left[a_{n}^{\prime} \mid k\right]$ are all zero. Thus $N\left(a_{n}, b_{n} ; l\right)=N\left(a_{n}^{\prime}, b_{n}^{\prime} ; l\right)$.

Finally, let us observe that the term $-2 b_{L} l^{2}$ is unchanged in the formula for $R$ when we replace $a_{n}$ by $a_{n}^{\prime}$. We have already noted the $b_{i}^{*}$ remain unchanged. Thus we just have to compare the differences $1 / \alpha-b_{n}^{*} / a_{n}$ and $1 /\left(\alpha+p b_{n}^{2}\right)-$ $b_{n}^{\prime *} /\left(a_{n}+p b_{n}\right)$. From the argument above $b_{n}^{\prime *}=b_{n}^{*}+m p$ where $b_{n} b_{n}^{*}=m a_{n}+1$. Then

$$
\begin{aligned}
\frac{1}{\alpha+p b_{n}^{2}}-\frac{b_{n}^{\prime *}}{a_{n}+p b_{n}} & =\frac{\left(1-b_{n} b_{n}^{\prime *}\right)}{b_{n}\left(a_{n}+p b_{n}\right)}=\frac{-m\left(a_{n}+p b_{n}\right)}{b_{n}\left(a_{n}+p b_{n}\right)} \\
& =\frac{-m}{b_{n}}=\frac{-m a_{n}}{b_{n} a_{n}}=\frac{1-b_{n} b_{n}^{*}}{b_{n} a_{n}}=\frac{1}{\alpha}-\frac{b_{n}^{*}}{a_{n}} .
\end{aligned}
$$

This completes the proof that

$$
R\left(a_{1}, \ldots, a_{n} ; l\right)=R\left(a_{1}, \ldots, a_{n-1}, a_{n}+p a_{1} \cdots a_{n-1} ; l\right) \text { for } l<a_{n} .
$$

These indices do differ when $l>a_{n}$. Calculations suggest $R\left(a_{1}, \ldots, a_{n} ; l\right) \geqslant$ $R\left(a_{1}, \ldots, a_{n-1}, a_{n}+p a_{1} \cdots a_{n-1}\right)$ for all $l$.

We conclude from this that if $R$ is positive for a particular Euler class for one term in the family, it will be positive for succeeding terms as long as $l<a_{n}$. If $l^{2} / a<4 / \max \left(a_{i}\right)$, the positivity gives a contradiction to bounding a positive definite manifold with no 2-torsion in $H_{1}$-in short, $M$ is non-PD bounding (cf. [FL, Corollary 1]). When $l^{2} / \alpha>4 / \max \left(a_{i}\right)$, the analysis becomes more delicate as one also has to check whether an invariant $\rho_{i}\left(b_{i}, 1 ; l\right)>l^{2} / \alpha$ for all $i=1, \ldots, n$ (cf. [FL, Corollary 2]). If not (in which case we will call the bundle determined by $l$ reducible) then we cannot get a contradiction even if $R>0$. (Reducibility can vary over members of the family even though $R$ is constant. Note that the range where we need not check for reducibility is constant $\left(l^{2} / b_{n}<4\right)$ if $a_{n}=\max \left(a_{i}\right)$ but varies if $a_{n} \neq \max \left(a_{i}\right)$.)

As an example, consider $M(3,14,37)$. For $l^{2} / 42<4, \quad R$ is negative. $R(3,14,37 ; 13)=1$ but the bundle is reducible. However, $R(3,14,37 ; 16)=1$ and $\rho_{i}(42,1 ; 16)>256 / 1554$ for all $i$ and so we can conclude $M(3,14,37)$ is non-PD bounding. What readers may be most interested in, however, is seeing why a given 
Brieskorn variety cannot bound an acyclic (or contractible) manifold. For $M(3,14,37)$, this could be seen using the mu invariant $\mu \neq 0$. If one wanted an example with $\mu=0$, the natural place to look would be the next member $M(3,14,79)$ of the family since the mu invariant alternates on this family and so $\mu(3,14,79)=0$. Unfortunately, for each $l$ with $R>0$ (e.g. $l=13,16$ ) the bundle is reducible. However, one can form the family $M(3,14+237 p, 79)$ on which $\mu=0$. Here $13<14$ implies $R(3,251,79 ; 13)=R(3,14,79 ; 13)=R(3,14,37 ; 13)=1$ and $169 / 237<4$ so we do not have to check for reducibility. Thus $M(3,251,79)$ has mu invariant 0 and is non-PD bounding.

REMARK. We note the behavior of the mu invariant on a family. For notational convenience we assume $a_{n}<b n=a_{1} \cdots a_{n-1}$, and denote the family as $M_{k}=$ $\left(a_{1}, \ldots, a_{n-1}, a_{n}+(k-1) b_{n}\right)$. Then $\mu\left(M_{k}\right)=\mu\left(M_{k+2}\right)$, cf. Neumann [N, Theorem 5.1].

\section{REFERENCES}

[FL] R. Fintushel and T. Lawson, Compactness of moduli spaces for orbifold instantons, Topology Appl. (to appear).

[FS1] R. Fintushel and R. Stern, Pseudofree orbifolds, Ann. of Math. (2) 122 (1985), 335-364.

[FS2] __ $O(2)$ actions on the 5-sphere, Invent. Math. (to appear).

[L] T. Lawson, Representing homology classes on almost definite 4-manifolds, Michigan Math. J. (to appear).

[N] W. Neumann, An invariant of plumbed homology spheres, Topology Symposium, Siegen, 1979, Lecture Notes in Math., vol. 788, Springer-Verlag, Berlin and New York, 1979, pp. 125-144.

[NZ] W. Neumann and D. Zagier, A Note on an invariant of Fintushel and Stern, Geometry and Topology (Proceedings, Univ. of Maryland, 1983-84), Lecture Notes in Math., vol. 1167, Springer-Verlag, Berlin and New York, 1985, pp. 241-244.

Department of Mathematics, Tulane University, New Orleans, Louisiana 70118 